\title{
MULTIFACILITY LOCATION PROBLEMS ON A SPHERE
}

\author{
TURCUT AYKIN \\ Division of Industrial Engineering \\ Alfred University, Alfred, New York 14802 \\ and

\section{A.J.G. BABU} \\ Division of Industrial and Management Systems Engineering
} University of South Florida, Tampa, Florida 33620

(Received September 3, 1986)

ABSTRACT. A unified approach to multisource location problems on a sphere is presented. Euclidean, squared Euclidean and the great circle distances are considered. An algorithm is formulated and its convergence properties are investigated.

KEY WORDS AND PHRASES. Facility location, optimum location, nonlinear programming, applications of mathematical programing.

1980 AMS SUBJECT CLASSIFICATION CODES. 90C30, $90 \mathrm{C} 50$.

\section{INTRODUCTION .}

The multifacility location problem can be defined as finding the location of new facilities, in a specified space, with respect to several existing facilities so that the sum of weighted distances between the locations of new facilities, and new and existing facilities is minimized. Mathematically, problem can be stated as:

$\underset{x_{1}, x_{2}, \ldots, x_{n} \in E_{n}}{\operatorname{minimize}} f\left(x_{1}, x_{2}, \ldots, x_{n}\right)=\sum_{1 \leq j<k \leq n} v_{j k} d\left(x_{j}, x_{k}\right)+\sum_{j=1}^{n} \sum_{1=1}^{m} w_{j 1} d\left(x_{j}, P_{1}\right)$

where $v_{j k}$ is a positive weight associated with new facilities $g$ and $k$, $w_{j l}$ is a positive weight associated with the existing facility 1 and new facility $\mathrm{f}$, for all 1 and $j, x_{j}=\left(x_{j 1}, x_{j 2}, \ldots, x_{j n}\right)^{\prime}$ is the location of the new facllity $f$ to be determined, $P_{1}=\left(p_{11}, p_{12}, \ldots, p_{1 n}\right)^{\prime}$ is the location of the existing facility 1 , and $d($.$) is the distance between two points by a given norm.$

Problem (1.1) has been treated extensively in the literature. The distance functions most commonly encountered are the Euclidean and rectilinear distances. Euclidean distance problem is discussed in [1-5]. Problem (1.1) involving rectilinear distance is studied in $[1,2],[6-9]$.

When modelling and analyzing the problem of finding the best geographical locations for a number of new facllities, an analyst has to make several assumptions. One of these assumptions is concerned with the size of the area covering the destinations (or the demand points). If the area covering the demand points is 
sufficiently small, then this part of the earth's surface can be approximated by a plane. And the problem can be analyzed and solved by using the well known techniques of location theory. However, the earth's surface can be described, with considerable exactness, as a sphere. And plane is a good approximation only for relatively small areas. When the destination points are widely separated, the area covering these points can no longer be approximated by a plane and (1.1) is no longer a suitable model. In this case the destination points and the new facilities are restricted to be on the sphere $\quad\|x\|^{2}=R, X \in E_{3}$, where $R$ is the radius of the earth's surface. Because of the constraint, solution space becomes nonconvex. And the shortest travel distance between two points is not the Euclidean distance but the geodesic (or great circle) distance. Problems concerning location of international headquarters, distribution/marketing centers, detection station placement, placement of radio transmitters for long range communication may fall into this category.

In recent years, many papers have dealt with the large region location problems. Location problems involving points on a sphere are discussed in [10-27]. A complete review of the state of art in spherical location can be found in Wesolowsky [25]. The only attempts to solve multisource location problems on a sphere, of which we are aware are due to Dhar and Rao [21-22]. They considered the multisource location problem on a sphere involving geodesic distance, and developed a normalized gradient method.

In this paper, we present a unified approach for the solution of multisource location problems on a sphere. Three distance measures are considered; Euclidean, squared Euclidean, and the great circle distance. An algorithm analogous to the Weiszfeld algorithm [28] for the classical Weber problem is formulated. When the distance measure is Euclidean or the squared Euclidean distance, it is shown that the proposed algorithm always converges to a local minimum. Computational results are presented.

2. PROBLEM FORMULATION.

Let $X$, and $Y$ be two points on the surface of a sphere (without loss of generality, we assume a sphere with unit radius, i.e. $R=1$ ). We consider the following metrics:

(1) Squared Euclidean distance : $d_{1}(X, Y)=\|X-Y\|^{2}$

(2) Euclidean distance : $\mathrm{d}_{2}(X, Y)=\|X-Y\|$

(3) Geodesic distance : $d_{3}(X, Y)=2 \arcsin \left(\frac{\| X-Y||}{2}\right)$

Note that the metrics are all of the form $d(X, Y)=h(\|X-Y\|)$. Using this general form the multifacility location problem on a sphere may be stated as follows:

$$
\begin{aligned}
& \min f\left(x_{1}, x_{2}, \ldots, x_{n}\right)=\sum_{1 \leq j<k \leq n} v_{j k} h\left(\left\|x_{j}-x_{k}\right\|\right)+\sum_{j=1}^{n} \sum_{i=1}^{m} w_{j i} h\left(\left\|x_{j}-P_{j}\right\|\right) \\
& \text { subject to } \\
& \qquad x_{j} \|^{2}=1, j=1,2, \ldots, n \\
& \quad x_{j} \in E_{3}, j=1,2, \ldots, n
\end{aligned}
$$

The objective function is bounded both from below and above and there exists a maximum as well as a minimum (Aykin and Babu [26]). Furthermore the solution space is not convex resulting in a non-convex programming problem. 


\section{THE ALGORITHM.}

In order to derive an algorithm we first derive necessary conditions for a set of points to be minimum. Using Lagrange multipliers $\mu_{j}, j=1,2 \ldots, n$, the following Lagrange function is obtained:

$$
\begin{aligned}
& L\left(x_{1}, x_{2}, \ldots, x_{n}\right)=\sum_{1 \leq j<k \leq n} v_{j k} h\left(\left\|x_{j}-x_{k}\right\|\right)+\sum_{j=1}^{n} \sum_{i=1}^{m} w_{j i} h\left(\left\|x_{j}-P_{j}\right\|\right) \\
& +\sum_{j=1}^{n} \mu_{j}\left(\left\|x_{j}\right\|^{2}-1\right)
\end{aligned}
$$

which leads to the necessary conditions

$$
\begin{aligned}
\nabla_{j} L=\sum_{\substack{k=1 \\
\neq j}}^{n} v_{j k} \frac{h^{\prime}\left(\left\|x_{j}-x_{k}\right\|\right)}{\left\|x_{j}-x_{j k}\right\|}\left(x_{j}-x_{k}\right)+\sum_{i=1}^{m} \frac{h^{\prime}\left(\left\|x_{j}-P_{f}\right\|\right)}{\left\|x_{j}-P_{f}\right\|}\left(x_{j}-P_{i}\right) \\
+2 \mu_{j} x_{j}=0, j=1,2, \ldots, n
\end{aligned}
$$

$\frac{\partial L}{\partial \mu_{j}}=\left\|x_{j}\right\|^{2}-1=0$

$, j=1,2, \ldots, n$

where $\nabla_{j} L$ is the vector of partial derivatives of $L\left(x_{1}, x_{2}, \ldots, x_{n}\right)$ with respect to $x_{j}, y_{j}, z_{j}(x, y, z$ coordinates of source $j)$. Solution of (3.2) and (3.3) yields

$$
\begin{aligned}
\left\{\sum_{\substack{k=1 \\
\neq j}}^{n} v_{j k} \frac{h^{\prime}\left(\mid x_{j}-x_{k} \|\right)}{\left\|x_{j}-x_{k}\right\|}+\sum_{i=1}^{m} w_{j i} \frac{h^{\prime}\left(\left\|x_{j}-P_{j}\right\|\right)}{\left\|x_{j}-P_{j}\right\|}+2 \mu_{j}\right\}^{2}\left\|x_{j}\right\|^{2} \\
=\left\|\sum_{\substack{k=1 \\
\neq j}}^{n} v_{j k} \frac{h^{\prime}\left(\left\|x_{j}-x_{k}\right\|\right)}{\left\|x_{j}-x_{k}\right\|} x_{k}+\sum_{i=1}^{m} w_{j 1} \frac{h^{\prime}\left(\left\|x_{j}-P_{j}\right\|\right)}{\left\|x_{j}-P_{j}\right\|} P_{i}\right\|^{2} \\
, j=1,2, \ldots, n
\end{aligned}
$$

so that

$$
\begin{aligned}
& x_{j}=T_{j}\left(x_{1}, x_{2}, \ldots, x_{n}\right)=\frac{\sum_{\substack{k=1 \\
\neq j}}^{n} v_{j k} \frac{h^{\prime}\left(\left\|x_{j}-x_{k l}\right\|\right)}{\left\|x_{j}-x_{k}\right\|} x_{k}+\sum_{i=1}^{m} \frac{h^{\prime}\left(\left\|x_{j}-P_{j}\right\|\right)}{\left\|x_{j}-P_{f}\right\|} P_{i}}{\mp\left\|\sum_{\substack{k=1 \\
\neq j}}^{n} v_{j k} \frac{h^{\prime}\left(\left\|x_{j}-x_{k}\right\|\right)}{\left\|x_{j}-x_{k}\right\|} x_{k}+\sum_{i=1}^{m} \frac{h^{\prime}\left(\left\|x_{j}-P_{1}\right\|\right)}{\left\|x_{j}-P_{1}\right\|} P_{f}\right\|} \\
& , j=1,2, \ldots, n
\end{aligned}
$$

By choosing positive sign in $(3.5)$ and by taking $T_{f}\left(x_{1}, x_{2}, \ldots, x_{n}\right), j=1,2, \ldots n$, as iteration functions an algorithm, analogous to Weiszfeld [28] algorithm, is defined as follows: 
Step 1. Designate the starting points by $x_{j}^{1}, j=1,2 \ldots, n$. Set $t=1$, and $\nu$.

Step 2. Calculate $x_{j}^{t+1}, j=1,2, \ldots, n$ by (3.5) using $x_{1}^{t+1}, x_{2}^{t+1}, \ldots, x_{j-1}^{t+1}$ $x_{g}^{t}, \ldots, x_{n}^{t}$, i.e. $x_{j}^{t+1}=T_{j}\left(x_{1}^{t+1}, x_{2}^{t+1}, \ldots, x_{j-1}^{t+1}, x_{j}^{t}, \ldots, x_{n}^{t}\right)$

Step 3. If $\max \left\{\left\|x_{j}^{t+1}-x_{j}^{t}\right\|, j=1,2, \ldots, n\right\} \leq \nu$ stop. Otherwise, set $t=t+1$ and go to step 2 .

In the algorithm given above, each source location is updated during the iteration in the sense that $x_{j}^{t+1}$ is a function of $x_{1}^{t+1}, x_{2}^{t+1}, \ldots, x_{j-1}^{t+1}, x_{j}^{t}, \ldots, x_{n}^{t}$ for all $j$. This approach is known as Gauss-Seidel procedure. An alternative, and potentially less efficient procedure, is to compute $x_{j}^{t+1}$ as a function of $x_{1}^{t}, x_{2}^{t}, \ldots, x_{j-1}^{t}, x_{j}^{t}, \ldots, x_{n}^{t}$, i.e. $x_{g}^{t+1}=r_{j}\left(x_{1}^{t}, x_{2}^{t}, \ldots, x_{n}^{t}\right)$. Note that if $\sum_{\substack{k=1 \\ \neq j}}^{n} v_{j k} \frac{h^{\prime}\left(\left\|x_{j}-x_{k}\right\|\right)}{\left\|x_{j}-x_{k}\right\|} x_{k}+\sum_{i=1}^{m} w_{j i} \frac{h^{\prime}\left(\left\|x_{j}-P_{i}\right\|\right)}{\left\|x_{j}-P_{i}\right\|} P_{i}=0$ for some $j$, then the iterative functions given in (3.5) will be undefined. We call such a set of vectors an irregular solution.

The algorithm defined by the iteration functions $T_{j}, j=1,2, \ldots, n$, gives a gradient projection method with precalculated step size. In this respect this algorithm is analogous to the Weiszfeld scheme [28]. Let $\nabla_{f} f$ be the vector of partial derivatives of $f$ with respect to $x_{j}, y_{j}, z_{j}\left(x, y, z\right.$ coordinates of $g^{\text {th }}$ source), then

$$
\nabla_{j} f=\sum_{\substack{k=1 \\ \neq j}}^{n} v_{j k} \frac{h^{\prime}\left(\left\|x_{j}-x_{k}\right\|\right)}{\left\|x_{j}-x_{k}\right\|}\left(x_{j}-x_{k}\right)+\sum_{i=1}^{m} w_{j i} \frac{h^{\prime}\left(\left\|x_{j}-P_{i}\right\|\right)}{\left\|x_{j}-P_{f}\right\|}\left(x_{j}-P_{i}\right)
$$

so that

$$
\sum_{\substack{k=1 \\ \neq j}}^{n} v_{j k} \frac{h^{\prime}\left(\left\|x_{j}-x_{k}\right\|\right)}{\left\|x_{j}-x_{k}\right\|} x_{k}+\sum_{i=1}^{m} w_{j i} \frac{h^{\prime}\left(\left\|x_{j}-P_{i}\right\|\right)}{\left\|x_{j}-P_{j}\right\|} P_{i}
$$




$$
\begin{array}{r}
=\left\{\sum_{\substack{k=1 \\
\neq j}}^{n} v_{j k} \frac{h^{\prime}\left(\mid x_{j}-x_{k} \|\right)}{\left\|x_{j}-x_{k}\right\|}+\sum_{i=1}^{m} w_{j i} \frac{h^{\prime}\left(\left\|x_{j}-P_{j}\right\|\right)}{\left\|x_{j}-P_{i}\right\|}\right\} x_{j}-\nabla_{j} f \\
, j=1,2, \ldots, n
\end{array}
$$

Therefore

$$
T_{j}\left(x_{1}, x_{2}, \ldots, x_{n}\right)=\frac{x_{j}-s_{j} \nabla_{j} f}{\left\|x_{j}-s_{j} \nabla_{j} f\right\|} \quad g=1,2, \ldots, n
$$

where

$$
\begin{aligned}
& s_{j}\left(x_{1}, x_{2}, \ldots, x_{n}\right)=1 /\left\{\sum_{\substack{k \neq 1 \\
\neq j}}^{n} v_{j k} \frac{h^{\prime}\left(\left\|x_{j}-x_{k}\right\|\right)}{\left\|x_{j}-x_{k}\right\|}+\sum_{i=1}^{m} v_{j 1} \frac{h^{\prime}\left(\left\|x_{j}-P_{1}\right\|\right)}{\left\|x_{j}-P_{1}\right\|}\right\} \\
& \text { 1) Squared Euclidean Distance }
\end{aligned}
$$

In this case $h(U)=U^{2}$ and $h^{\prime}(U)=2 U$. Then (3.5) becomes

$$
x_{j}=T_{j}\left(x_{1}, x_{2}, \ldots, x_{n}\right)=\frac{\sum_{\substack{k=1 \\ \neq j}}^{n} v_{j k} x_{k}+\sum_{i=1}^{m} w_{j 1} P_{i}}{\left\|\sum_{\substack{k=1 \\ \neq j}}^{n} v_{j k} x_{k}+\sum_{i=1}^{m} w_{j 1} P_{i}\right\|} \quad, j=1,2, \ldots n
$$

One useful property of the algorithm proposed is that the objective function value decreases at each iteration. This is proved in the following theorem.

THEOREM 1. Suppose $\underline{X}=\left\{x_{1}, X_{2}, \ldots, X_{n}\right\}$ is a regular solution. Let $T(x)=$ $\left\{\mathrm{T}_{1}(\mathrm{X}), \mathrm{T}_{2}(\mathrm{X}), \ldots, \mathrm{T}_{\mathrm{n}} \underline{(\mathrm{X})}\right\}$ be the mapping given in (3.10) then the mapping $\mathrm{T}(\mathrm{X})$ is a descent mapping; that is $f(T(\underline{X})) \leq f(\underline{X})$ and $f(T(\underline{X}))=f(\underline{X})$ if $\underline{X}=T(\underline{X})$.

PROOF. Let $\underline{x}^{t}=\left\{x_{1}^{t}, x_{2}^{t}, \ldots, x_{n}^{t}\right\}$ be the set of the current source locations at the end of $t^{\text {th }}$ 1teration. Value of the objective function at $\underline{x}^{t}$ is $f\left(x_{1}^{t}, x_{2}^{t}, \ldots, x_{n}^{t}\right)$. Let $x_{1}^{t+1}=T_{1}\left(x_{1}^{t}, x_{2}^{t}, \ldots, x_{n}^{t}\right)$. Now consider another problem, which is a single facillty location problem with the squared Euclidean distance, and with the existing facilities $x_{2}^{t}, \ldots, x_{n}^{t}, P_{1}, P_{2}, \ldots, P_{m}\left(1 . e\right.$. sources $x_{2}, \ldots, x_{n}$ are "fixed" at their current locations).

minimize $s_{1}\left(x_{1}\right)=\sum_{j=2}^{n} v_{1 j}\left\|x_{1}-x_{j}\right\|^{2}+\sum_{1=1}^{m} w_{11}\left\|x_{1}-P_{1}\right\|^{2}+s_{1}\left(x_{2}^{t}, \ldots, x_{n}^{t}\right)$

subject to

$$
\begin{gathered}
\left\|x_{1}\right\|^{2}=1, \\
x_{1} \in E_{3}
\end{gathered}
$$


where

$$
s_{1}\left(x_{2}^{t}, \ldots, x_{n}^{t}\right)=\sum_{2 \leq j<k \leq n} v_{j k}\left\|x_{j}^{t}-x_{k}^{t}\right\|^{2}+\sum_{j=2}^{n} \sum_{i=1}^{m} w_{j i}\left\|x_{j}^{t}-P_{1}\right\|^{2}
$$

Since the sources $x_{j}, j=2, \ldots, n$ are "fixed" at their current locations $g_{1}\left(x_{2}^{t}, \ldots, x_{n}^{t}\right)$ is is a constant and therefore does not affect the optimum solution of this problem.

Spherical single facility location problem with the squared Euclidean distance was first formulated and shown to have a unique minimum by Katz and Cooper [14]. They formulated this problem as to

minimize $f(X)=\sum_{i=1}^{m} w_{i}\left\|X-P_{f}\right\|^{2}$

subject to

$$
\begin{aligned}
& \|x\|^{2}=1, \\
& x \in E_{3}
\end{aligned}
$$

and showed that the unique optimizing location is

$$
x=\frac{\sum_{i=1}^{m} w_{i} P_{1}}{\left\|\sum_{i=1}^{m} w_{i} P_{1}\right\|}
$$

Hence the minimizing location for the problem (3.11) is given by

$$
x_{1}^{t+1}=\frac{\sum_{j=2}^{n} v_{1 j} x_{j}^{t}+\sum_{i=1}^{m} w_{1 i} p_{1}}{\left\|\sum_{j=2}^{n} v_{1 j} x_{j}^{t}+\sum_{i=1}^{m} w_{1 i} p_{i}\right\|}
$$

Note that (3.15) is equivalent to(3.10) for $j=1$. Since $x_{1}^{t+1}$ is the minimizing solution to the problem (3.11) if $x_{1}^{t+1} \neq x_{1}^{t}$, then $f_{1}\left(x_{1}^{t+1}\right)<f_{1}\left(x_{1}^{t}\right)$. But $f_{1}\left(x_{1}\right)=$ $f\left(x_{1}, x_{2}^{t}, \ldots, x_{n}^{t}\right)$. Thus we obtain the following inequality:

$$
f\left(x_{1}^{t+1}, x_{2}^{t}, \ldots, x_{n}^{t}\right)<f\left(x_{1}^{t}, x_{2}^{t}, \ldots, x_{n}^{t}\right)
$$

And $f\left(x_{1}^{t+1}, x_{2}^{t}, \ldots, x_{n}^{t}\right)=f\left(x_{1}^{t}, x_{2}^{t}, \ldots, x_{n}^{t}\right)$ if and only if $x_{1}^{t+1}=x_{1}^{t}$, or $x_{1}=T_{1}\left(x_{1}, x_{2}^{t}, \ldots, x_{n}^{t}\right)$.

Now let $x_{2}^{t+1}=T_{2}\left(x_{1}^{t+1}, x_{2}^{t}, \ldots . x_{n}^{t}\right)$. By using the same analogy, we formulate another problem which is again a single facility location problem with the squared Euclidean distance and the existing facilities $x_{1}^{t+1}, x_{3}^{t}, \ldots, x_{n}^{t}, P_{1}, \ldots, P_{m}$, 1.e. now the sources $x_{1}$, 
and $x_{j}, f=3, \ldots, n$, are "fixed" at their current locations.

$$
\text { minimize } \begin{aligned}
f_{2}\left(x_{2}\right)= & v_{21}\left\|x_{2}-x_{1}^{t+1}\right\|^{2}+\sum_{j=3}^{n} v_{2 j}\left\|x_{2}-x_{j}^{t}\right\|^{2} \\
& +\sum_{i=1}^{m} w_{2 i}\left\|x_{2}-P_{j}\right\|^{2}+s_{2}\left(x_{1}^{t+1}, x_{3}^{t}, \ldots, x_{n}^{t}\right)
\end{aligned}
$$

subject to

$$
\underset{x_{2} \in E_{3}}{\left\|x_{2}\right\|^{2}=1}
$$

where

$$
\begin{aligned}
g_{2}\left(x_{1}^{t+1}, x_{3}^{t}, \ldots, x_{n}^{t}\right)= & \sum_{3 \leq j<k \leq n} v_{j k}\left\|x_{j}^{t}-x_{k}^{t}\right\|^{2}+\sum_{j=3}^{n} v_{1 j}\left\|x_{1}^{t+1}-x_{j}^{t}\right\|^{2} \\
& +\sum_{j=3} \sum_{i=1}^{m} w_{j 1}\left\|x_{j}^{t}-P_{1}\right\|^{2} \\
& +\sum_{i=1}^{m} w_{1 i}\left\|x_{1}^{t+1}-P_{1}\right\|^{2}
\end{aligned}
$$

is a constant. Again this problem has a unique solution.

$$
x_{2}^{t+1}=\frac{\sum_{j=3}^{n} v_{2 j} x_{j}^{t}+v_{12} x_{1}^{t+1}+\sum_{i=1}^{m} w_{21} P_{1}}{\left\|\sum_{j=3}^{n} v_{2 j} x_{j}^{t}+v_{12} x_{1}^{t+1}+\sum_{i=1}^{m} w_{21} P_{1}\right\|}
$$

This is equivalent to $(3.10)$ for $y=2$. Since $x_{2}^{t+1}$ is the minimizing location for the problem (3.17), we have the following inequality:

$$
f_{2}\left(x_{2}^{t+1}\right)<f_{2}\left(x_{2}^{t}\right)
$$

provided that $x_{2}^{t+1} \neq x_{2}^{t}$. But $f_{2}\left(x_{2}\right)=f\left(x_{1}^{t+1}, x_{2}, x_{3}^{t}, \ldots, x_{n}^{t}\right)$. Thus

$$
f\left(x_{1}^{t+1}, x_{2}^{t+1}, x_{3}^{t}, \ldots, x_{n}^{t}\right)<e\left(x_{1}^{t+1}, x_{2}^{t}, x_{3}^{t}, \ldots, x_{n}^{t}\right)
$$

By combining the inequalities (3.16) and (3.21), we obtain 


$$
f\left(x_{1}^{t+1}, x_{2}^{t+1}, x_{3}^{t}, \ldots, x_{n}^{t}\right)<f\left(x_{1}^{t}, x_{2}^{t}, x_{3}^{t}, \ldots, x_{n}^{t}\right)
$$

provided that $x_{1}^{t+1} \neq x_{1}^{t}$ and/or $x_{2}^{t+1} \neq x_{2}^{t}$. By repeating the same steps for $x_{j}, j=3, \ldots, n$, we get

$$
f\left(x_{1}^{t+1}, x_{2}^{t+1}, \ldots, x_{n}^{t+1}\right)<f\left(x_{1}^{t}, x_{2}^{t}, \ldots, x_{n}^{t}\right)
$$

whereas $\quad f\left(x_{1}^{t+1}, x_{2}^{t+1}, \ldots, x_{n}^{t+1}\right)=f\left(x_{1}^{t}, x_{2}^{t}, \ldots, x_{n}^{t}\right)$ if and only if $\left\{x_{1}^{t+1}, x_{2}^{t+1}, \ldots, x_{n}^{t+1}\right\}$ $=\left\{x_{1}^{t}, x_{2}^{t}, \ldots, x_{n}^{t}\right\}$, or $\underline{x}=T(\underline{x})$.

Thus when the metric is the squared Euclidean distance the objective function value decreases at each iteration, and the algorithm either converges to a minimum or stops at an irregular solution.

COROLLARY 1. Suppose $\left\{x_{1}, x_{2}, \ldots, x_{n}\right\}$ is a regular solution. If $x_{j}, j=1,2, \ldots, n$, is the minimizing location with respect to both the other new facilities and the destination points then the solution is a minimizing solution to the problem.

PROOF. Proof follows from Theorem 1.

Note that the method used above can be easily extended to other location problems. In order to prove that the algorithm designed for the solution of a multifacility location problem is a descent algorithm, it is sufficient to prove the descent mapping property of the same algorithm for the single facility version of the same problem.

2) Euclidean Distance

In this case $h(U)=U$ and $h^{\prime}(U)=1$. (3.5) then becomes

$$
x_{j}=T_{j}\left(x_{1}, x_{2}, \ldots, x_{n}\right)=\frac{\sum_{\substack{k=1 \\ \neq j}}^{n} \frac{v_{j k} x_{k}}{\left\|x_{j}-x_{k}\right\|}+\sum_{i=1}^{m} \frac{w_{j i} P_{i}}{\left\|x_{j}-P_{j}\right\|}}{\left\|\sum_{k=1}^{n} \frac{v_{j k} x_{k}}{\left\|x_{j}-x_{k}\right\|}+\sum_{i=1}^{m} \frac{w_{j i} P_{i}}{\left\|x_{j}-P_{i}\right\|}\right\|} \quad j=1,2, \ldots, n
$$

The iteration functions given in (3.24) are not defined at all the points in the solution space. In particular, if either new facilities $j$ and $k$ have the same location or new facility $j$ and existing facility $i$ have the same location then the objective function $f\left(x_{1}, x_{2}, \ldots, x_{n}\right)$ is not differentiable and, therefore, the iteration functions (3.24) are not defined. In order to resolve this difficulty, the Hyperbolic Approximation Procedure of Eyster et al [2] is used as follows:

$$
d(X, Y)=\left[\|X-Y\|^{2}+\xi\right]^{1 / 2}
$$

where $\xi$ isoa small positive constant.

We now prove that the iteration functions (3.24) define a descent mapping in the following theorem.

THOREM 2. Suppose $\underline{x}=\left\{x_{1}, x_{2}, \ldots, x_{n}\right\}$ is a regular solution, $x_{j} \neq x_{k}$ for $j \neq k$, and $X_{j} \neq P_{i}$ for all $\mathrm{j}$, i. Let $T \underline{(x)}=\left\{T_{1} \underline{(X)}, T_{2} \underline{(X)}, \ldots, T_{n} \underline{(X)}\right\}$ be the mapping given in (3.24), then the mapping $T(\underline{X})$ is a descent mapping. 
PROOF. Let $\underline{x}=\left\{x_{1}^{t}, x_{2}^{t}, \ldots, x_{n}^{t}\right\}$ be the set of the current source locations, and $x_{j}^{t+1}=T_{j}\left(x_{1}^{t+1}, x_{2}^{t+1}, \ldots, x_{j-1}^{t+1}, x_{j}^{t}, \ldots, x_{n}^{t}\right), j=1,2, \ldots, n$. Now consider the following problem.

$$
\begin{aligned}
\operatorname{minimize} \mathrm{f}_{j}\left(\mathrm{x}_{j}\right)=\sum_{\mathrm{k}=1}^{j-1} v_{j k}\left\|\mathrm{x}_{j}-\mathrm{x}_{k}^{t+1}\right\| & +\sum_{k=j+1}^{n} v_{j k}\left\|\mathrm{x}_{j}-\mathrm{x}_{k}^{t}\right\| \\
& +g_{j}\left(x_{1}^{t+1}, x_{2}^{t+1}, \ldots, x_{j=1}^{t+1}, x_{j+1}^{t}, \ldots, x_{n}^{t}\right)
\end{aligned}
$$

subject to

$$
\begin{aligned}
& \left\|x_{j}\right\|^{2}=1, \\
& x_{j} \in E_{3}
\end{aligned}
$$

where

$$
\begin{aligned}
g_{j}\left(x_{1}^{t+1}, x_{2}^{t+1}, \ldots, x_{j-1}^{t+1}, x_{j+1}^{t}, \ldots, x_{n}^{t}\right)=\sum_{1 \leq q<k \leq j-1} v_{q k}\left\|x_{q}^{t+1}-x_{k}^{t+1}\right\| \\
+\sum_{q=1}^{j-1} \sum_{k=j+1}^{n} v_{q k}\left\|x_{q}^{t+1}-x_{k}^{t}\right\|+\sum_{j+1 \leq q<k \leq n} v_{q k}\left\|x_{q}^{t}-x_{k}^{t}\right\| \\
+\sum_{k=1}^{j-1} \sum_{i=1}^{m} w_{k i}\left\|x_{k}^{t+1}-P_{i}\right\|+\sum_{k=j+1}^{n} \sum_{i=1}^{m} w_{k i}\left\|x_{k}^{t}-P_{1}\right\|
\end{aligned}
$$

is a constant. Note that this problem is a single facility location problem with the existing facilities $x_{1}^{t+1}, x_{2}^{t+1}, \ldots, x_{j-1}^{t+1}, x_{j+1}^{t}, \ldots, x_{n}^{t}, P_{1}, P_{2}, \ldots, P_{m}$, for $\mathrm{j}=1,2, \ldots, n$.

Problem (3.13) involving Euclidean distance has been studied by Katz and Cooper [14]. They proposed a descent algorithm with the following iterative function.

$$
x=\frac{\sum_{i=1}^{m} \frac{w_{1} P_{1}}{\left\|x-P_{1}\right\|}}{\left\|\sum_{i=1}^{m} \frac{w_{i} P_{i}}{\left\|x-P_{1}\right\|}\right\|}
$$

This iterative function can be used iteratively to solve (3.26) as follows.

$$
x_{j}^{t+1}=\frac{\sum_{k=1}^{j-1} \frac{v_{j k} x_{k}^{t+1}}{\left\|x_{j}^{t}-x_{k}^{t+1}\right\|}+\sum_{k=j+1}^{n} \frac{v_{j k} x_{k}^{t}}{\left\|x_{j}^{t}-x_{k}^{t}\right\|}+\sum_{i=1}^{m} \frac{w_{j i} P_{1}}{\left\|x_{j}^{t}-P_{1}\right\|}}{\left\|\sum_{k=1}^{j-1} \frac{v_{j k} x_{k}^{t+1}}{\left\|x_{j}^{t}-x_{k}^{t+1}\right\|}+\sum_{k=j+1}^{n} \frac{v_{j k} x_{k}^{t}}{\left\|x_{j}^{t}-x_{k}^{t}\right\|}+\sum_{i=1}^{m} \frac{w_{j 1} P_{1}}{\left\|x_{j}^{t}-P_{1}\right\|}\right\|}
$$

Since (3.28) defines a descent mapping, we have 


$$
f_{j}\left(x_{j}^{t+1}\right)<f_{j}\left(x_{j}^{t}\right)
$$

if $x_{j}^{t+1} \neq x_{j}^{t}$. But $f_{j}\left(x_{j}\right)=f\left(x_{1}^{t+1}, x_{2}^{t+1}, \ldots, x_{j-1}^{t+1}, x_{j}, x_{j+1}^{t}, \ldots x_{n}^{t}\right) . \quad$ (3.30) then becomes

$$
\begin{aligned}
f\left(x_{1}^{t+1}, x_{2}^{t+1}, \ldots, x_{j-1}^{t+1},\right. & \left.x_{j}^{t+1}, x_{j+1}^{t}, \ldots, x_{n}^{t}\right) \\
& <f\left(x_{1}^{t+1}, x_{2}^{t+1}, \ldots, x_{j-1}^{t+1}, x_{j}^{t}, x_{j+1}^{t}, \ldots, x_{n}^{t}\right)
\end{aligned}
$$

By repeating the same steps for $x_{j}, j=1,2, \ldots, n$, we get

$$
f\left(x_{1}^{t+1}, x_{2}^{t+1}, \ldots, x_{n}^{t+1}\right) \leq f\left(x_{1}^{t}, x_{2}^{t}, \ldots, x_{n}^{t}\right)
$$

whereas $f\left(x_{1}^{t+1}, x_{2}^{t+1}, \ldots, x_{n}^{t+1}\right)=f\left(x_{1}^{t}, x_{2}^{t}, \ldots, x_{n}^{t}\right)$ if $\left\{x_{1}^{t+1}, x_{2}^{t+1}, \ldots, x_{n}^{t+1}\right\}=\left\{x_{1}^{t}, x_{2}^{t}, \ldots, x_{n}^{t}\right\}$, 1.e. $\underline{X}=T(\underline{x})$.

Thus when the metric is the Euclidean distance, the objective function value decreases at each iteration, and the algorithm either converges to a local minimu, or stops at an irregular solution, or when $x_{j}=x_{k}, j \neq k$, or $x_{j}=P_{i}$, for any $j, i$.

3) Geodesic Distance

In this case $h(U)=2 \arcsin (U / 2), U=|X-Y| \mid=[2(1-X Y)]^{1 / 2}$, and

$$
\begin{aligned}
& \frac{h^{\prime}(\|X-Y\|)}{\|X-Y\|}=\frac{1}{\left.\mid 1-(X Y)^{2}\right]^{1 / 2}} \cdot \text { (3.5) then becomes } \\
& x_{j}=T_{j}\left(x_{1}, x_{2}, \ldots, x_{n}\right)=\frac{\sum_{\substack{k=1 \\
\neq j}}^{n} \frac{v_{j k} x_{k}}{\left[1-\left(x_{j} x_{k}\right)^{2}\right]^{1 / 2}}+\sum_{i=1}^{m} \frac{w_{j i} P_{1}}{\left[1-\left(x_{j} P_{i}\right)^{2}\right]^{172}}}{\left\|\sum_{\substack{k=1 \\
\neq j}}^{n} \frac{v_{j k} x_{k}}{\left[1-\left(x_{j} x_{k}\right)^{2}\right]^{1 / 2}}+\sum_{i=1}^{m} \frac{w_{j i} P_{i}}{\left[1-\left(x_{j} P_{i}\right)^{2}\right]^{1 / 2}}\right\|} \\
& , j=1,2 \ldots, n
\end{aligned}
$$

An iterative scheme for determining minimizing source locations $x_{j}, f=1,2, \ldots, n$, is now defined by taking (3.33) as iterative functions and using them in the algorithm outlined before.

Note that the iterative functions (3.33), and the partial derivatives of the objective function $f\left(x_{1}, x_{2}, \ldots, x_{n}\right)$ are not defined when $x_{j}=x_{k}$ for $j \neq k$, or $x_{j}=P_{i}$ for any $j, i$, or when two new facilities $j$ and $k$, or new facility $j$ and existing facillty 1 have the coordinates of $a$ point and 1 ts antipode ( $X$ and $Y$ on a unit sphere are antipodal points if $\left.d_{3}(X, Y)=\pi\right)$. In order to resolve this difficulty, we used the Hyperbolic Approximation Procedure of Eyster et al [2].

We have not succeeded in proving that the algorithm with (3.33) as the iteration functions is a descent algorithm. However, above in Theorems 1, and 2, we proved that the algorithms with $(3.10)$ and $(3.24)$ as the iteration functions are descent algorithms. In an analogous manner, one may prove that the algorithm proposed is a descent algorithm. 
4. AN EXAMPLE PROBLEM.

This problem is concerned with the location of three distribution centers. The products are to be distributed to 10 European and Asian cities by air. The names and the location coordinates of the cities, and the weights are shown in Tables 1 , and 2 .

We solved this problem using the proposed algorithm with $\xi=10^{-12}$ and, $\nu=$ 0.00001 starting from $x_{1}=\left(0 ., 0 ., 1_{.}\right)^{\prime}, x_{2}=\left(0 ., 0 ., 1_{.}\right)^{\prime}$, and $x_{3}=\left(0 ., 0_{., 1 .}\right)^{\prime}$. The results are presented in Table 3. Additionally this problem was run 10 times with random starting points and the algorithm always converged to a unique solution. Although the difference between the optimal objective function values, calculated using geodesic distances, is small, the distance between the optimum source locations is significant.

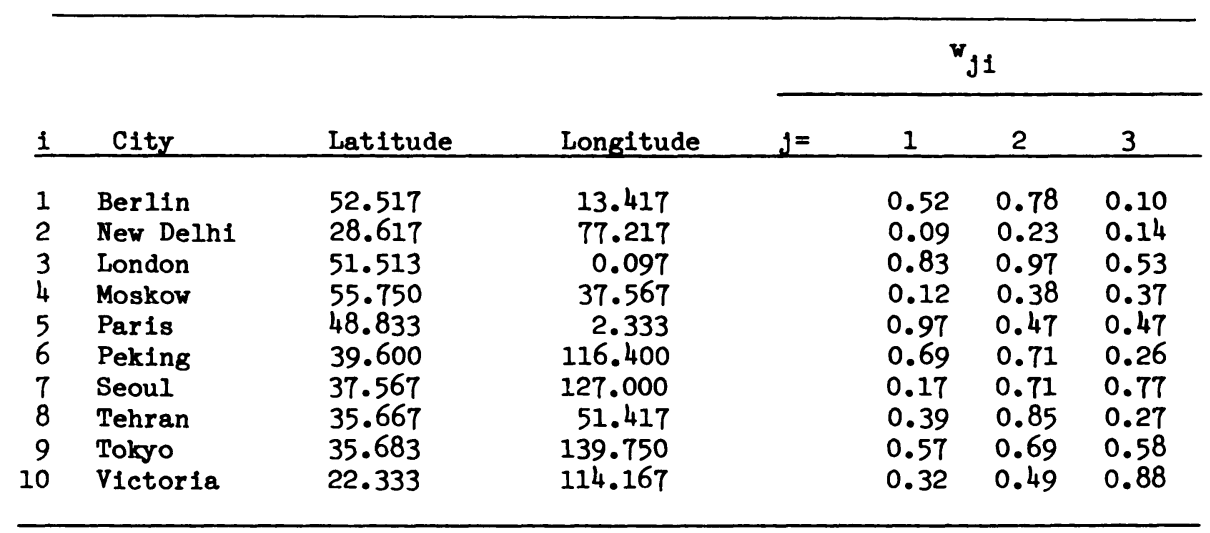

TABLE 1. Weights and Locations of Existing Facilities

\begin{tabular}{ll|llll}
\hline $\mathbf{v}_{\text {jk }}$ & $\mathrm{j}$ & $\mathrm{k}=$ & 1 & 2 & 3 \\
\hline & & 0.0 & 0.15 & 0.25 \\
2 & & 0.15 & 0.0 & 0.25 \\
3 & & 0.25 & 0.25 & 0.0 \\
\hline
\end{tabular}

TABLE 2. Weights, $\mathbf{v}_{j k}$

\begin{tabular}{lcccc}
\hline Measure & Stops at (Latitude, Longitude) & Obj.Fn.Val. No. of Iter. \\
\hline $\begin{array}{l}\text { Squared } \\
\text { Euclidean }\end{array}$ & $(59.042,62.591),(55.580,73.663),(52.207,90.548)$ & 571.434 & 5 \\
Euclidean & $(53.017,13.911),(54.736,52.394),(40.655,114.890)$ & 569.608 & 102 \\
Geodesic & $(56.745,37.356),(54.521,59.743),(45.620,104.939)$ & 565.164 & 61 \\
\hline
\end{tabular}


1. DREZNER, Z. and WESOLOWSKY, G.O. A Trajectory Method for the Optimization of the Multi-Facility Location Problem with Ip Distances, Management Science 24 $(1978), 238-261$.

2. EYSTER,J.W., WHITE, J.A. and WIERWILLE, W.W. On Solving Multifacility Location Problems Using a Hyperboloid Approximation Procedure, AIIE Trans. 5 (1973), 1-6.

3. FRANCIS, R.L. and CABOT, A.V. Properties of a Multifacility Location Problem Involving Euclidean Distance, Nav. Res. Logist. Q. 19 (1972), 335-353.

4. VERGIN, R.C. and ROGERS, J.D. An Algorithm and Computational Procedure for Locating Economic Facilities, Management Science 13 (1967), 240-254.

5. LOVE, R.F. Locating Facilities in Three-Dimensional Space by Convex Programming, Nav. Res. Logist. Q. 16 (1969), 503-516.

6. CABOT,A.V., FRANCIS, R.L. and STARY, M.A. A Network Flow Solution to a Rectilinear Distance Facility Location Problem, AIIE Trans. ㄴ (1970), 132-141.

7. FRANCIS, R.L. On the Location of Multiple New Facilities with Respect to Existing Facilities, J. of Industrial Engineering 15 (1964), 106-107.

8. WESOLOWSKY, G.O. and LOVE,R.F. The Optimal Location of New Facilities Using Rectangular Distances, Operations Research 19 (1971), 124-130.

9. WESOLOWSKY, G.O. and LOVE, R.F. A Nonlinear Approximation Method for Solving a Generalized Rectangular Distance Weber Problem, Management Science 18 (1972), 56-63.

10. ZAMBO, J. Optimum Location of Mining Facilities, Akademiai Kiado, Budapest, 1968.

11. WENDELL, R.E. Some Aspects in the Theory of Location, Ph. D. Dissertation, Northwestern University, Evanston, Illinois, 1971.

12. KATZ, I.N. and COOPER, L. Optimum Location on a Sphere, Paper Presented at the Joint ORSA/TIMS National Meeting, Chicago, 1975.

13. KATZ, I.N. and COOPER, L. Optimum Location on a Sphere, Paper Presented at the Joint ORSA/TIMS National Meeting, Ph1ladelphia, 1976.

14. KATZ, I.N. and COOPER, L. Optimum Location on a Sphere, Comput. and Math. with Appl. 6 (1980), 175-196.

15. DREZNER, Z. and WESOLOWSKY, G.0. Facility Location on a Sphere, J. Opl. Res. Soc. $\underline{29}(1978), 997-1004$.

16. DREZNER, Z. and WESOLOWSKY, G.O. Minimax and Maximin Facility Location Problems on a Sphere, Nav. Res. Logist. Q. 30 (1983), 305-312.

17. LITWHILER, D.W. and ALY, A.A. Large Region Location Problems, Computers and Operations Research 6 (1979), 1-12.

18. DREZNER, Z. On Location Dominance on Spherical Surface, Operations Research 29 $(1981), 1218-1219$.

19. DREZNER, Z. A Solution of the Weber Location Problem on a Sphere, J. Opl. Res. Soc. 36 (1985), 333-334. 
20. DHAR, U.R. and RAO, J.R. A Comparative Study of Three Norms for Facility Location Problem on Spherical Surface, New Zeland Journal of Operations Research 8 (1980), 173-183.

21. DHAR, U.R. and RAO, J.R. On Solving Multi-Source Location Problem on a Sphere, Scientific Management on Transportation Systems, (Ed. N.K. Jaiswal and B.K. Bener J1), North Holland Publishing Co., Amsterdam, 1980.

22. DHAR, U.R. and RAO, J.R. Domain Approximation Method for Solving Multifacility Location Problems on a Sphere, J. Opl. Res. Soc. 33 (1982), 639-645.

23. DHAR, U.R. and RAO, J.R. An Efficient Algorithm for Solving Area-Constrained Location Problems on a Sphere, Opsearch 19 (1982), 23-32.

24. DREZNER, Z. Constrained Location Problems in the Plane and on a Sphere, AIIE Trans. 15 (1983), 300-304.

25. WESOLOWSKY, G.0. Location Problems on a Sphere, Reg. Sc1. Urban Econ. 12 (1983), 495-508.

26. AYKIN, T. and BABU, A.J.G. The Weber Problem on a Surface, Paper Presented at the Joint ORSA/TIMS National Meeting, Orlando, 1983.

27. AYKIN, T. and BABU, A.J.G. Constrained Large Region Location Problems - I: Single Facility Location, Paper Presented at the Joint ORSA/TIMS National Meeting, San Francisco, 1984.

28. WEISZFELD, E. Surle Point Pour Lequel La Somme des Distances de n Points Donnes est Minimum, Tohoku Math. J. 43 (1937), 335-386.

29. BAZARAA, M.S. and SHETTY, C.M. Nonlinear Programming: Theory and Algorithms, John Wiley and Sons, Inc., New York, 1979.

30. KHITROV, B.F. On the Application of Various Curves to the Solution of Geodesic Problems on an Ellipsoid, Geodesy, Mapping, and Photogrammetry 15 (1973), 97-101. 


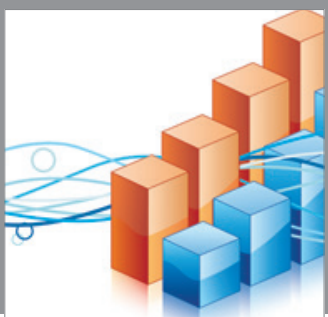

Advances in

Operations Research

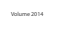

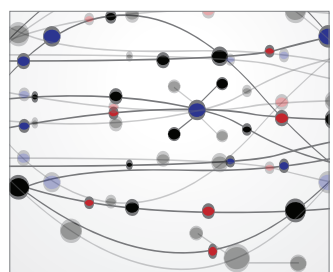

\section{The Scientific} World Journal
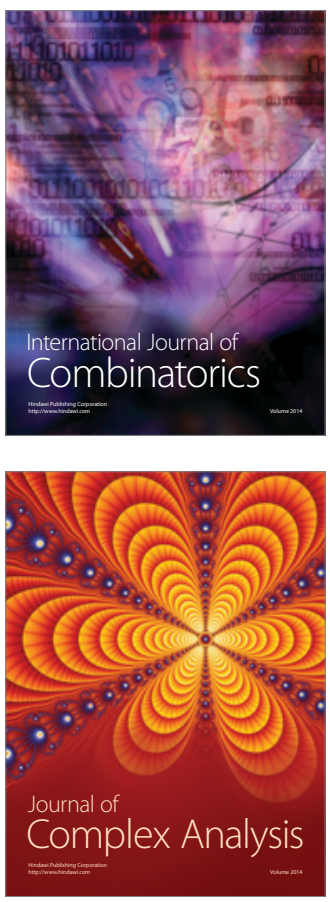

International Journal of

Mathematics and

Mathematical

Sciences
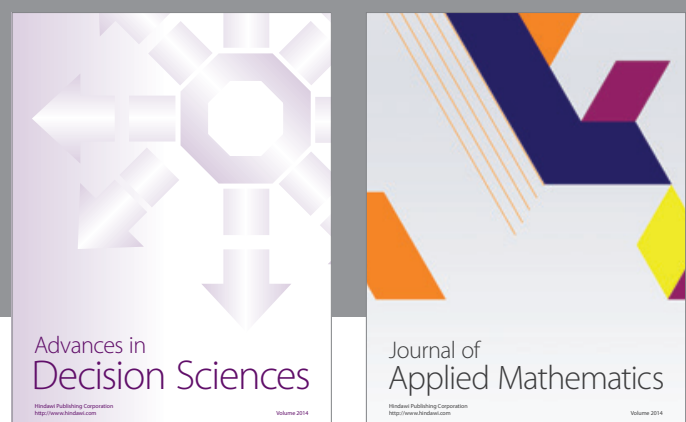

Journal of

Applied Mathematics
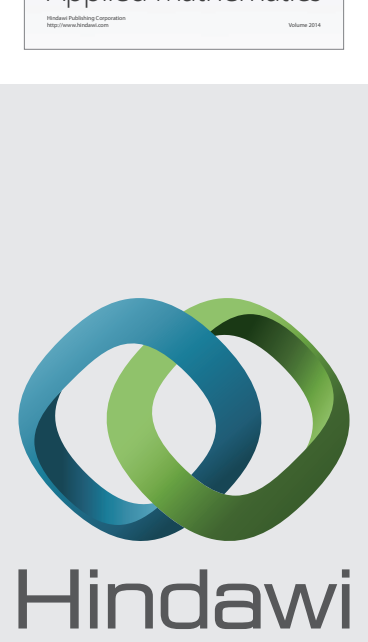

Submit your manuscripts at http://www.hindawi.com
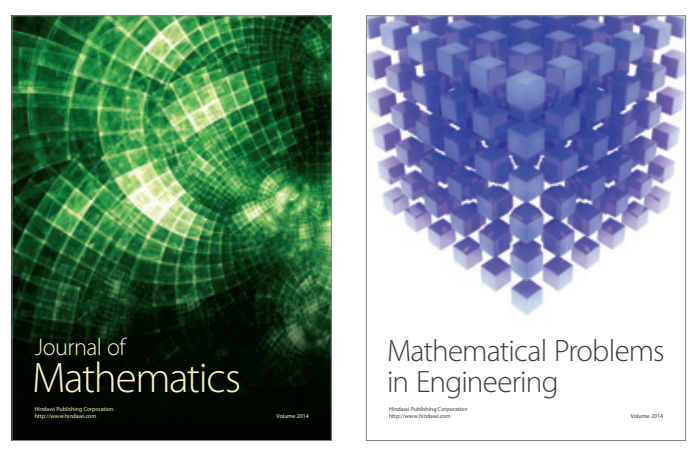

Mathematical Problems in Engineering
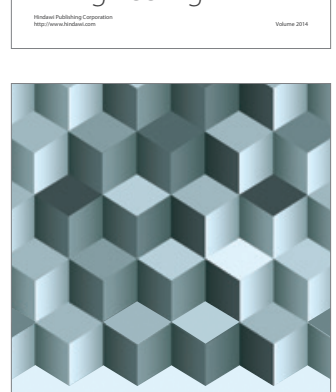

Journal of

Function Spaces
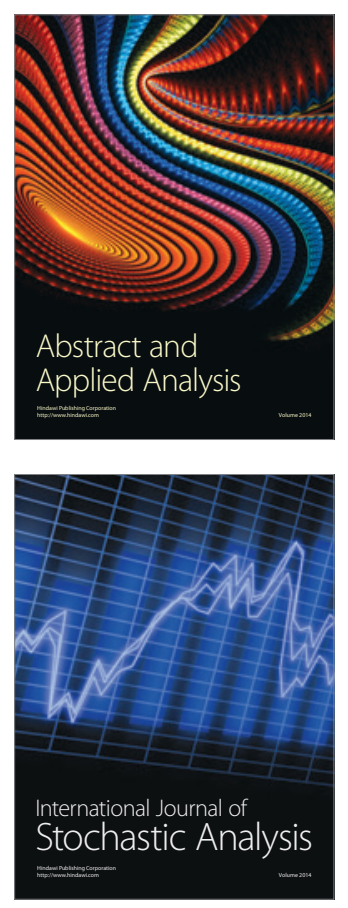

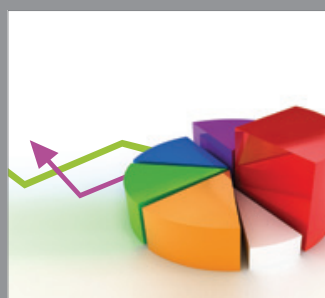

ournal of

Probability and Statistics

Promensencen
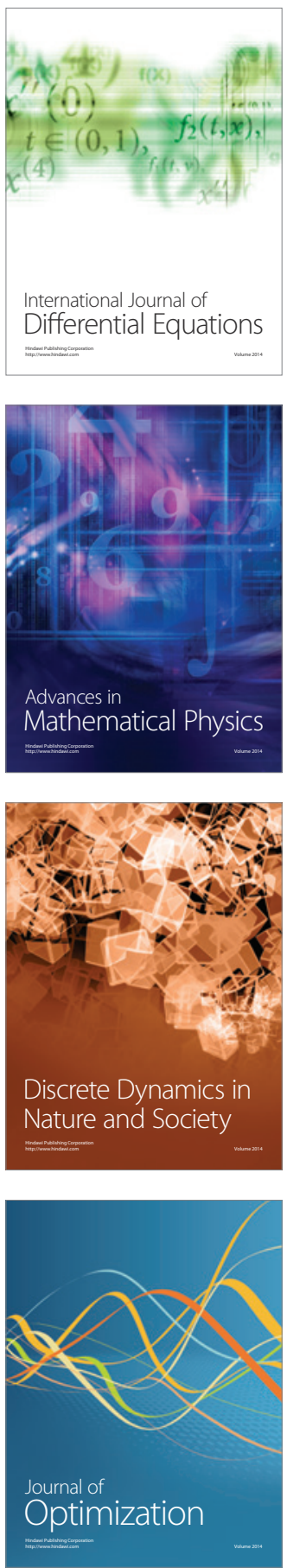